Noname manuscript No.

(will be inserted by the editor)

\title{
Stability and Stabilization of 2D Singular Systems: A Strict LMI Approach
}

\author{
Marwa Elloumi • Mariem Ghamgui . \\ Driss Mehdi • Fernando Tadeo • \\ Mohamed Chaabane
}

Received: date / Accepted: date

\begin{abstract}
This paper deals with the stability and stabilization of 2D singular systems described by a Roesser model. The proposed results are presented in terms of a strict Linear Matrix Inequality (LMI), which makes possible to elaborate a new sufficient admissibility condition. Based on this condition the design of a state feedback controller is also treated, deriving a sufficient condition for the admissibility of the closed loop system. A numerical example is given at the end of the paper to illustrate the effectiveness of the proposed methodology.
\end{abstract}

Keywords Two-Dimensional (2D) Systems · Singular Systems · Stability • Stabilization · Strict LMI

Marwa Elloumi

National School of Engineers of Sfax, University of Sfax, Sfax, 3038, Tunisia

E-mail: marwa.elloumi91@gmail.com

Mariem Ghamgui

National School of Engineers of Sfax, University of Sfax, Sfax, 3038, Tunisia

National School of Engineering of Poitiers, University of Poitiers, Poitiers, 86022, France

E-mail: mariemghamgui@yahoo.fr

Driss Mehdi

National School of Engineering of Poitiers, University of Poitiers, Poitiers, 86022, France E-mail: driss.mehdi@univ-poitiers.fr

Fernando Tadeo

Industrial Engineering School, University of Valladolid, 47005 Valladolid, Spain

E-mail: Fernando.Tadeo@uva.es

Mohamed Chaabane

National School of Engineers of Sfax, University of Sfax, Sfax, 3038, Tunisia

E-mail: chaabane.ucpi@gmail.com 


\section{Introduction}

Two-dimensional (2D) systems are attracting significant attention from the research community, due to the presence of this class of systems in water stream heating, seismographic data processing, thermal processes, multidimensional digital filtering, process control, image and signal processing, etc $[13,16,15$, 17]. Among the relevant results already published, the stability of $2 \mathrm{D}$ systems has been studied in, for example, [10,12,21,23]; the stabilization of $2 \mathrm{D}$ systems has been explored in $[25,9,18,11]$.

This paper concentrates on $2 \mathrm{D}$ singular systems which have been studied in the few past decades and which could have various practical applications in many areas such as: digital filtering, modeling and control of electrical circuits, power systems, economics etc. They describe physical systems that involve algebraic non-dynamic constraints $[8,6]$.

In practical $2 \mathrm{D}$ system one can consider some algebraic constraints on the state vector components (an example is given for $1 \mathrm{D}$ system in [7]). The description becomes then the one of a $2 \mathrm{D}$ singular system. It is worth noting that singularity may also be a result of modelling $[1,2]$.

The jump behavior and the associated stability analysis for 2D singular systems was established in [29]. The stability of $2 \mathrm{D}$ singular systems has been further studied in, for example, [3,31,24]. Other problems like $H_{\infty} 2 \mathrm{D}$ filtering, $H_{\infty} 2 \mathrm{D}$ control and $H_{\infty} 2 \mathrm{D}$ model reduction have been reported in $[14,27,26]$. Most of these works are developed based on non-strict LMI which motivates us to search for stability and stabilization conditions in terms of a strict LMI. Some previous results obtained for $2 \mathrm{D}$ singular systems extend fundamental results on $1 \mathrm{D}$ singular systems $[28,20,5]$. For example, the admissibility and state feedback admissibilization of discrete singular systems have been elaborated in [19] and the stabilization of continuous descriptor systems has been investigated in [4].

Most of the published works in the literature use z-transformation approach and algebraic methods. Here, decomposition methods are used as already performed for 1D Singular systems and we extend these techniques to 2D singular deterministic Systems.

In this paper, we then study the admissibility of $2 \mathrm{D}$ singular systems described by a Roesser model using a strict LMI, that is tractable using available LMI software solvers. This result is used to derive a controller that ensures the admissibility of the closed loop.

We mainly focus for the time being on theoretical development but we hope in the near future be able to deal with some application in the discretization of partial differential equation which are already modeled as 2D systems.

The structure of this paper is as follows: Section 2 gives the problem formulation. In section 3, we discuss the regularity and causality of $2 \mathrm{D}$ singular systems described by a Roesser model. In section 4, we establish the stability of this class of system, expressed by means of a strict LMI. In section 5 , we deal with the problem of stabilization by state feedback for these systems. Section 6 presents a numerical example to highlight the usefulness of the proposed 
method.

\section{Notations}

In this paper, the following notations will be used:

* Sym $\{A\}$ stands for the addition of a matrix and its symmetric:

$$
\operatorname{Sym}\{A\}=A^{T}+A \text {. }
$$

$* \operatorname{diag}\{$.$\} represents a block-diagonal matrix.$

$*$ For simplicity, instead of $X^{\top} Y X$, we will write $(\bullet)^{\top} Y X$ where $(\bullet)$ replaces $X . X$ and $Y$ are some matrix expressions.

\section{Problem Formulation}

The class of $2 \mathrm{D}$ discrete systems studied in this paper is described by the following Singular Roesser Model (SRM):

$$
E\left[\begin{array}{l}
x^{h}(i+1, j) \\
x^{v}(i, j+1)
\end{array}\right]=A\left[\begin{array}{l}
x^{h}(i, j) \\
x^{v}(i, j)
\end{array}\right]
$$

where $x^{h} \in \mathbb{R}^{n_{h}}$ is the horizontal state vector, $x^{v} \in \mathbb{R}^{n_{v}}$ is the vertical state vector, $A \in \mathbb{R}^{n \times n}$, with $n=n_{h}+n_{v}$ is the system matrix.

\section{Definition 1 [27]}

(i) The system (1) is said to be stable if $\lambda(E, A) \subset D_{\text {int }}(0,1)$.

(ii) The system (1) is said to be admissible if it is regular, causal and stable.

Definition 2 [30]

A 2D Singular Roesser Model is said to be of the separated standard form if it is of the form (1) with

$$
E=\operatorname{diag}\left\{E^{h}, E^{v}\right\}
$$

where $E_{h} \in \mathbb{R}^{n_{h} \times n_{h}}$ and $E_{v} \in \mathbb{R}^{n_{v} \times n_{v}}$.

For system described by (1), for now on matrix $E$ will comply with (2).

\section{Regularity and causality of $2 \mathrm{D}$ singular systems}

For a singular system, a solution can be ensured to exist only if the system is regular, that is, if the system can be transformed into standard subsystems, with an algebraic constraint on the state vector of the system.

If the matrix $A$ is partitioned as follows:

$$
A=\left[\begin{array}{ll}
A^{h h} & A^{h v} \\
A^{v h} & A^{v v}
\end{array}\right]
$$


Then, one can write the 2D SRM equivalently as follows:

$$
\begin{aligned}
E^{h} x^{h}(i+1, j) & =A^{h h} x^{h}(i, j)+A^{h v} x^{v}(i, j) \\
E^{v} x^{v}(i, j+1) & =A^{v h} x^{h}(i, j)+A^{v v} x^{v}(i, j)
\end{aligned}
$$

with $\operatorname{rank}\left(E^{k}\right)=r^{k}$, for $k=h, v$.

Let us consider the matrices $U^{k}$ and $V^{k}$, for $k=h, v$ such that

$$
U^{k} E^{k} V^{k}=\left[\begin{array}{cc}
I_{r^{k}} & 0 \\
0 & 0
\end{array}\right]
$$

with the state vectors partitioned similarly:

$$
\bar{x}^{k}=\left(V^{k}\right)^{-1} x^{k}=\left[\begin{array}{l}
\bar{x}_{1}^{k} \\
\bar{x}_{2}^{k}
\end{array}\right]
$$

where $\bar{x}_{1}^{k}$ is in $\mathbb{R}^{r^{k}}$ and $\bar{x}_{2}^{k}$ is in $\mathbb{R}^{\left(n^{k}-r^{k}\right)}$, for $k=h, v$.

Let also:

$$
\bar{A}^{k l}=U^{k} A^{k l} V^{l}, \quad k, l=h, v .
$$

Multiplying the left side of equation (4) by $U^{h}$ we get

$$
\begin{aligned}
U^{h} E^{h} V^{h}\left(V^{h}\right)^{-1} x^{h}(i+1, j) & =U^{h} A^{h h} V^{h}\left(V^{h}\right)^{-1} x^{h}(i, j)+ \\
& U^{h} A^{h v} V^{v}\left(V^{v}\right)^{-1} x^{v}(i, j) \\
& =\bar{A}^{h h} \bar{x}^{h}(i, j)+\bar{A}^{h v} \bar{x}^{v}(i, j)
\end{aligned}
$$

$$
\begin{aligned}
{\left[\begin{array}{rr}
I_{r^{h}} & 0 \\
0 & 0
\end{array}\right] \bar{x}^{h}(i+1, j) } & =\left[\begin{array}{c}
\bar{x}_{1}^{h}(i+1, j) \\
0
\end{array}\right] \\
& =\left[\begin{array}{c}
\bar{A}_{11}^{h h} \bar{x}_{1}^{h}(i, j)+\bar{A}_{12}^{h h} \bar{x}_{2}^{h}(i, j)+\bar{A}_{11}^{h v} \bar{x}_{1}^{v}(i, j)+\bar{A}_{12}^{h v} \bar{x}_{2}^{v}(i, j) \\
\bar{A}_{21}^{h h} \bar{x}_{1}^{h}(i, j)+\bar{A}_{22}^{h h} \bar{x}_{2}^{h}(i, j)+\bar{A}_{21}^{h v} \bar{x}_{1}^{v}(i, j)+\bar{A}_{22}^{h v} \bar{x}_{2}^{v}(i, j)
\end{array}\right]
\end{aligned}
$$

which can be written as follows

$$
\begin{aligned}
\bar{x}_{1}^{h}(i+1, j) & =\bar{A}_{11}^{h h} \bar{x}_{1}^{h}(i, j)+\bar{A}_{12}^{h h} \bar{x}_{2}^{h}(i, j)+\bar{A}_{11}^{h v} \bar{x}_{1}^{v}(i, j)+\bar{A}_{12}^{h v} \bar{x}_{2}^{v}(i, j) \\
0 & =\bar{A}_{21}^{h h} \bar{x}_{1}^{h}(i, j)+\bar{A}_{22}^{h h} \bar{x}_{2}^{h}(i, j)+\bar{A}_{21}^{h v} \bar{x}_{1}^{v}(i, j)+\bar{A}_{22}^{h v} \bar{x}_{2}^{v}(i, j) .
\end{aligned}
$$

Performing the same operation for the second equation (4), we get

$$
\begin{aligned}
U^{v} E^{v} V^{v}\left(V^{v}\right)^{-1} x^{v}(i, j+1)= & U^{v} A^{v h} V^{h}\left(V^{h}\right)^{-1} x^{h}(i, j)+ \\
& U^{v} A^{v v} V^{v}\left(V^{v}\right)^{-1} x^{v}(i, j) \\
& =\bar{A}^{v h} \bar{x}^{h}(i, j)+\bar{A}^{v v} \bar{x}^{v}(i, j)
\end{aligned}
$$

$$
\begin{aligned}
{\left[\begin{array}{rr}
I_{r^{v}} & 0 \\
0 & 0
\end{array}\right] \bar{x}^{v}(i, j+1) } & =\left[\begin{array}{c}
\bar{x}_{1}^{v}(i, j+1) \\
0
\end{array}\right] \\
& =\left[\begin{array}{c}
\bar{A}_{11}^{v h} \bar{x}_{1}^{h}(i, j)+\bar{A}_{12}^{v h} \bar{x}_{2}^{h}(i, j)+\bar{A}_{11}^{v v} \bar{x}_{1}^{v}(i, j)+\bar{A}_{12}^{v v} \bar{x}_{2}^{v}(i, j) \\
\bar{A}_{21}^{v h} \bar{x}_{1}^{h}(i, j)+\bar{A}_{22}^{v h} \bar{x}_{2}^{h}(i, j)+\bar{A}_{21}^{v v} \bar{x}_{1}^{v}(i, j)+\bar{A}_{22}^{v v} \bar{x}_{2}^{v}(i, j)
\end{array}\right]
\end{aligned}
$$


and similarly as for the horizontal direction, we get

$$
\begin{aligned}
\bar{x}_{1}^{v}(i, j+1) & =\bar{A}_{11}^{v h} \bar{x}_{1}^{h}(i, j)+\bar{A}_{12}^{v h} \bar{x}_{2}^{h}(i, j)+\bar{A}_{11}^{v v} \bar{x}_{1}^{v}(i, j)+\bar{A}_{12}^{v v} \bar{x}_{2}^{v}(i, j) \\
0 & =\bar{A}_{21}^{v h} \bar{x}_{1}^{h}(i, j)+\bar{A}_{22}^{v h} \bar{x}_{2}^{h}(i, j)+\bar{A}_{21}^{v v} \bar{x}_{1}^{v}(i, j)+\bar{A}_{22}^{v v} \bar{x}_{2}^{v}(i, j)
\end{aligned}
$$

Equations (8) and (10) imply that

$$
\left[\begin{array}{ll}
\bar{A}_{21}^{h h} & \bar{A}_{21}^{h v} \\
\bar{A}_{21}^{v h} & \bar{A}_{21}^{v v}
\end{array}\right]\left[\begin{array}{l}
\bar{x}_{1}^{h}(i, j) \\
\bar{x}_{1}^{v}(i, j)
\end{array}\right]+\left[\begin{array}{ll}
\bar{A}_{22}^{h h} & \bar{A}_{22}^{h v} \\
\bar{A}_{22}^{v h} & \bar{A}_{22}^{v v}
\end{array}\right]\left[\begin{array}{l}
\bar{x}_{2}^{h}(i, j) \\
\bar{x}_{2}^{v}(i, j)
\end{array}\right]=0
$$

whereas equations (7) and (9) imply that

$$
\left[\begin{array}{l}
\bar{x}_{1}^{h}(i+1, j) \\
\bar{x}_{1}^{v}(i, j+1)
\end{array}\right]=\left[\begin{array}{cc}
\bar{A}_{11}^{h h} & \bar{A}_{11}^{h v} \\
\bar{A}_{11}^{v h} & \bar{A}_{11}^{v v}
\end{array}\right]\left[\begin{array}{l}
\bar{x}_{1}^{h}(i, j) \\
\bar{x}_{1}^{v}(i, j)
\end{array}\right]+\left[\begin{array}{ll}
\bar{A}_{12}^{h h} & \bar{A}_{12}^{h v} \\
\bar{A}_{12}^{v h} & \bar{A}_{12}^{v v}
\end{array}\right]\left[\begin{array}{l}
\bar{x}_{2}^{h}(i, j) \\
\bar{x}_{2}^{v}(i, j)
\end{array}\right]
$$

It comes then that if we assume that

$$
\left[\begin{array}{ll}
\bar{A}_{22}^{h h} & \bar{A}_{22}^{h v} \\
\bar{A}_{22}^{v h} & \bar{A}_{22}^{v v}
\end{array}\right] \text { is non singular }
$$

we get from (11)

$$
\left[\begin{array}{l}
\bar{x}_{2}^{h}(i, j) \\
\bar{x}_{2}^{v}(i, j)
\end{array}\right]=-\left[\begin{array}{ll}
\bar{A}_{22}^{h h} & \bar{A}_{22}^{h v} \\
\bar{A}_{22}^{v h} & \bar{A}_{22}^{v v}
\end{array}\right]^{-1}\left[\begin{array}{ll}
\bar{A}_{21}^{h h} & \bar{A}_{21}^{h v} \\
\bar{A}_{21}^{v h} & \bar{A}_{21}^{v v}
\end{array}\right]\left[\begin{array}{l}
\bar{x}_{1}^{h}(i, j) \\
\bar{x}_{1}^{v}(i, j)
\end{array}\right]
$$

Using (14) in (12), we get a standard 2D subsystem as follows

$$
\left[\begin{array}{l}
\bar{x}_{1}^{h}(i+1, j) \\
\bar{x}_{1}^{v}(i, j+1)
\end{array}\right]=\left(\overline{\bar{A}}_{11}-\overline{\bar{A}}_{12} \overline{\bar{A}}_{22}^{-1} \overline{\bar{A}}_{21}\right)\left[\begin{array}{l}
\bar{x}_{1}^{h}(i, j) \\
\bar{x}_{1}^{v}(i, j)
\end{array}\right]
$$

With:

$$
\begin{array}{ll}
\overline{\bar{A}}_{11}=\left[\begin{array}{ll}
\bar{A}_{11}^{h h} & \bar{A}_{11}^{h v} \\
\bar{A}_{11}^{v h} & \bar{A}_{11}^{v v}
\end{array}\right], & \overline{\bar{A}}_{12}=\left[\begin{array}{ll}
\bar{A}_{12}^{h h} & \bar{A}_{12}^{h v} \\
\bar{A}_{12}^{v h} & \bar{A}_{12}^{v v}
\end{array}\right], \\
\overline{\bar{A}}_{22}=\left[\begin{array}{ll}
\bar{A}_{22}^{h h} & \bar{A}_{22}^{h v} \\
\bar{A}_{22}^{v h} & \bar{A}_{22}^{v v}
\end{array}\right], & \overline{\bar{A}}_{21}=\left[\begin{array}{ll}
\bar{A}_{21}^{h h} & \bar{A}_{21}^{h v} \\
\bar{A}_{21}^{v h} & \bar{A}_{21}^{v v}
\end{array}\right]
\end{array}
$$

As a consequence, if condition (13) is satisfied, the system will be regular and causal. Moreover, if the matrix $\left(\overline{\bar{A}}_{11}-\overline{\bar{A}}_{12} \overline{\bar{A}}_{22}^{-1} \overline{\bar{A}}_{21}\right)$ is stable, then the system (1) is stable. This development is summarized by Theorem 1.

Theorem 1 If condition (13) is satisfied, then system (1) is regular and causal.

One can effectively check the admissibility of a $2 \mathrm{D}$ singular system by first checking the singularity of (13) and then the stability of (15). However, the use of Linear Matrix Inequalities (LMI) is more appropriate to solve such a problem, in particular if the system matrices are corrupted by uncertainties. In addition, the available LMI toolboxes are nowadays very efficient. That's why, it's more appropriate to work with this approach. In the case of a state feedback design, the state matrix is effectively $A+B K$. The objective in the design is to compute $K$ in such a way that the closed loop system is admissible, that is, regular causal and stable. In the design, condition (13) will depend on $K$. As a consequence, it will be difficult, if not impossible, to use (13) to check the non singularity and (15) for the stability. 


\section{Stability of $2 \mathrm{D}$ singular systems}

To state the first theorem on the stability of 2D singular, let us introduce some matrices that will be used in the sequel. Recall that $E$ and $A$ are defined as in (2) and (3). First, define the following permutation matrix

$$
J=\left[\begin{array}{cccc}
I_{r^{h}} & 0 & 0 & 0 \\
0 & 0 & I_{r^{v}} & 0 \\
0 & I_{n^{h}-r^{h}} & 0 & 0 \\
0 & 0 & 0 & I_{n^{v}-r^{v}}
\end{array}\right]
$$

satisfying $J J^{\top}=I$ and

$$
\begin{aligned}
& U=\left[\begin{array}{cc}
U^{h} & 0 \\
0 & U^{v}
\end{array}\right] \\
& V=\left[\begin{array}{cc}
V^{h} & 0 \\
0 & V^{v}
\end{array}\right]
\end{aligned}
$$

where $U^{h}, U^{v}, V^{h}$ and $V^{v}$ are defined by (6).

Theorem 2 If there exist symmetric matrices $P^{h}$ and $P^{v}$ and a matrix $Y$ such as the inequalities

$$
\begin{array}{r}
E P E^{\top}+\operatorname{Sym}\left\{E^{\dagger} Y\right\}>0 \\
A P A^{\top}-E P E^{\top}<0
\end{array}
$$

hold, with

$$
P=\left[\begin{array}{cc}
P^{h} & 0 \\
0 & P^{v}
\end{array}\right]
$$

and

$$
E^{\dagger}=(V U)^{-1}-E
$$

then system (1) is regular and stable.

Proof

Condition (17) makes possible to prove that the top left entry of $\overline{\bar{P}}=J V^{-1} P V^{-\top} J^{\top}$ is positive definite.

Indeed, multiplying (17) on the left by $J U$ and on the right by its transpose we get 


$$
\begin{aligned}
& J U E P E^{\top} U J^{\top}+\operatorname{Sym}\left\{J U E^{\dagger} Y U J^{\top}\right\} \\
& =J U E V J J^{\top} V^{-1} P V^{-\top} J J^{\top} V^{\top} E^{\top} U J^{\top}+\operatorname{Sym}\left\{\left(J U E^{\dagger} V J^{\top}\right) J V^{-1} Y U J^{\top}\right\} \\
& =\overline{\bar{E}} \overline{\bar{P}}^{\top} \overline{\bar{E}}^{\top}+\operatorname{Sym}\left\{(I-\overline{\bar{E}})\left[\begin{array}{ll}
\bar{Y}_{11} & \bar{Y}_{12} \\
\bar{Y}_{21} & \bar{Y}_{22}
\end{array}\right]\right\} \\
& =\left[\begin{array}{cc}
\overline{\bar{P}}_{11} & 0 \\
0 & 0
\end{array}\right]+\operatorname{Sym}\left\{\left(\left[\begin{array}{ll}
0 & 0 \\
0 & I
\end{array}\right]\right)\left[\begin{array}{ll}
\bar{Y}_{11} & \bar{Y}_{12} \\
\bar{Y}_{21} & \bar{Y}_{22}
\end{array}\right]\right\} \\
& =\left[\begin{array}{ll}
\overline{\bar{P}}_{11} & \bar{Y}_{21} \\
\bar{Y}_{21}^{\top} & \bar{Y}_{22}+\bar{Y}_{22}^{\top}
\end{array}\right]>0
\end{aligned}
$$

with: $\overline{\bar{E}}=\left[\begin{array}{ll}I & 0 \\ 0 & 0\end{array}\right]$.

It is easy to notice that (19) states effectively that $\overline{\bar{P}}_{11}>0$.

The second inequality makes possible to prove the regularity and the stability of the system under investigation.

Effectively, multiplying (18) by $J U$ on the left, and by its transpose on the right, we get

$$
J U A P A^{\top} U^{\top} J^{\top}-J U E P E^{\top} U^{\top} J^{\top}<0 .
$$

The second term in (20) can then be expressed as

$$
\begin{aligned}
J U E P E^{\top} U^{\top} J^{\top} & =\left(J U E V J^{\top}\right)\left(J V^{-1} P V^{-\top} J^{\top}\right)\left(J V^{\top} E^{\top} U^{\top} J^{\top}\right) \\
& =\left(J \bar{E} J^{\top}\right)\left(J \bar{P} J^{\top}\right)\left(J \bar{E}^{\top} J^{\top}\right)
\end{aligned}
$$

with:

$$
\begin{aligned}
& \bar{P}=V^{-1} P V^{-\top}=\left[\begin{array}{cc}
\bar{P}^{h} & 0 \\
0 & \bar{P}^{v}
\end{array}\right] \\
& \overline{\bar{P}}=J \bar{P} J^{\top}=\left[\begin{array}{cccc}
\bar{P}_{11}^{h} & 0 & \bar{P}_{12}^{h} & 0 \\
0 & \bar{P}_{11}^{v} & 0 & \bar{P}_{12}^{v} \\
\bar{P}_{21}^{h} & 0 & \bar{P}_{22}^{h} & 0 \\
0 & \bar{P}_{21}^{v} & 0 & \bar{P}_{22}^{v}
\end{array}\right]=\left[\begin{array}{cc}
\bar{P}_{11} & \overline{\bar{P}}_{12} \\
\bar{P}_{21} & \overline{\bar{P}}_{22}
\end{array}\right] \\
& \overline{\bar{E}}=J \bar{E} J^{-1}=\left[\begin{array}{cccc}
I_{r^{h}} & 0 & 0 & 0 \\
0 & I_{r^{v}} & 0 & 0 \\
0 & 0 & 0 & 0 \\
0 & 0 & 0 & 0
\end{array}\right]
\end{aligned}
$$

where $\bar{P}^{h}$ and $\bar{P}^{v}$ were partitioned similarly as, for instance, $\bar{A}^{h h}$.

The previous development implies that

$$
J U E P E^{\top} U^{\top} J^{\top}=\left[\begin{array}{cccc}
\bar{P}_{11}^{h} & 0 & 0 & 0 \\
0 & \bar{P}_{11}^{v} & 0 & 0 \\
0 & 0 & 0 & 0 \\
0 & 0 & 0 & 0
\end{array}\right]
$$


The first term of the Lyapunov inequality (18) can then be transformed as follows

$\left(J U A V J^{\top}\right)\left(J V^{-1} P V^{-\top} J^{\top}\right)\left(J V^{\top} A^{\top} U^{\top} J^{\top}\right)=\left(J \bar{A} J^{\top}\right)\left(J \bar{P} J^{\top}\right)\left(J \bar{A}^{\top} J^{\top}\right)$

with

$$
\bar{A}=U A V
$$

Moreover, defining

$$
\overline{\bar{A}}=J^{T} \bar{A} J=\left[\begin{array}{ll}
\overline{\bar{A}}_{11} & \overline{\bar{A}}_{12} \\
\bar{A}_{21} & \overline{\bar{A}}_{22}
\end{array}\right]
$$

and $T_{21}$ such as

$$
\left[\begin{array}{cr}
I & 0 \\
-\overline{\bar{P}}_{21} \overline{\bar{P}}_{11}^{-1} & I
\end{array}\right]\left[\begin{array}{cc}
\overline{\bar{P}}_{11} & \overline{\bar{P}}_{12} \\
\overline{\bar{P}}_{21} & \overline{\bar{P}}_{22}
\end{array}\right] \underbrace{\left[\begin{array}{cc}
I & -\overline{\bar{P}}_{11}^{-1} \overline{\bar{P}}_{12} \\
0 & I
\end{array}\right]}_{T_{21}}=\left[\begin{array}{cc}
\overline{\bar{P}}_{11} & 0 \\
0 & \overline{\bar{P}}_{22}-\overline{\bar{P}}_{21} \overline{\bar{P}}_{11}^{-1} \overline{\bar{P}}_{12}
\end{array}\right]
$$

with: $\overline{\bar{P}}_{21}=\overline{\bar{P}}_{12}^{T}$ and $\overline{\bar{P}}_{11}=\overline{\bar{P}}_{11}^{\top}$.

The first term of (18) can be transformed to give

$$
\begin{aligned}
& (\bullet)^{\top}\left(T_{21}^{\top} J \bar{P} J^{\top} T_{21}\right)\left(T_{21}^{-1} J \bar{A}^{\top} J^{\top}\right) \\
& =\left[\begin{array}{cc}
L_{11} & \bar{A}_{12} \\
L_{12} & \overline{\bar{A}}_{22}
\end{array}\right]\left[\begin{array}{cc}
\overline{\bar{P}}_{11} & 0 \\
0 & \hat{P}_{22}
\end{array}\right]\left[\begin{array}{cc}
L_{11}^{\top} & L_{12}^{\top} \\
\overline{\bar{A}}_{12}^{\top} & \overline{\bar{A}}_{22}^{\top}
\end{array}\right] \\
& =\left[\begin{array}{lll}
L_{11} \overline{\bar{P}}_{11} L_{11}^{\top}+\overline{\bar{A}}_{12} \hat{P}_{22} \overline{\bar{A}}_{21}^{\top} & L_{11} \overline{\bar{P}}_{11} L_{12}^{\top}+\overline{\bar{A}}_{12} \hat{P}_{22} \overline{\bar{A}}_{22}^{\top} \\
L_{12} \overline{\bar{P}}_{11} L_{11}^{\top}+\overline{\bar{A}}_{22} \hat{P}_{22} \overline{\bar{A}}_{21}^{\top} & L_{12} \overline{\bar{P}}_{11} L_{12}^{\top}+\overline{\bar{A}}_{22} \hat{P}_{22} \overline{\bar{A}}_{22}^{\top}
\end{array}\right]
\end{aligned}
$$

with:

$$
\begin{aligned}
& \hat{P}_{22}=\overline{\bar{P}}_{22}-\overline{\bar{P}}_{21} \overline{\bar{P}}_{11}^{-1} \overline{\bar{P}}_{12}, \\
& L_{11}=\left(\overline{\bar{A}}_{11}^{\top}+\overline{\bar{P}}_{11}^{-1} \overline{\bar{P}}_{12} \overline{\bar{A}}_{12}^{\top}\right)^{\top}, \\
& L_{12}=\left(\overline{\bar{A}}_{21}^{\top}+\overline{\bar{P}}_{11}^{-1} \overline{\bar{P}}_{12} \overline{\bar{A}}_{22}^{\top}\right)^{\top} .
\end{aligned}
$$

At this step, the Lyapunov inequality reads

$$
\left[\begin{array}{ll}
L_{11} \overline{\bar{P}}_{11} L_{11}^{\top}+\overline{\bar{A}}_{12} \hat{P}_{22} \overline{\bar{A}}_{21}^{\top} L_{11} \overline{\bar{P}}_{11} L_{12}^{\top}+\overline{\bar{A}}_{12} \hat{P}_{22} \overline{\bar{A}}_{22}^{\top} \\
L_{12} \overline{\bar{P}}_{11} L_{11}^{\top}+\overline{\bar{A}}_{22} \hat{P}_{22} \overline{\bar{A}}_{21}^{\top} L_{12} \overline{\bar{P}}_{11} L_{12}^{\top}+\overline{\bar{A}}_{22} \hat{P}_{22} \overline{\bar{A}}_{22}^{\top}
\end{array}\right]-\left[\begin{array}{cc}
\overline{\bar{P}}_{11} & 0 \\
0 & 0
\end{array}\right]<0
$$

from which we get

$$
L_{12} \overline{\bar{P}}_{11} L_{12}^{\top}+\overline{\bar{A}}_{22} \hat{P}_{22} \overline{\bar{A}}_{22}^{\top}<0 .
$$

Taking account of the fact that $\overline{\bar{P}}_{11}>0$, inequality (28) implies that $\overline{\bar{A}}_{22}$ is non singular.

Indeed, if $\overline{\bar{A}}_{22}$ is singular, then there exist $\xi \neq 0$ such as $\xi^{\top} \overline{\bar{A}}_{22}=0$. Thus, 
multipliying the left side of (28) by $\xi^{\top}$ and the right side by $\xi$, we get the following inequality

$$
\xi^{\top} L_{12} \overline{\bar{P}}_{11} L_{12}^{\top} \xi+\xi^{\top} \overline{\bar{A}}_{22} \hat{P}_{22} \overline{\bar{A}}_{22}^{\top} \xi<0
$$

Assume $L_{12}^{\top} \xi=a$, we get

$$
a^{\top} \overline{\bar{P}}_{11} a<0
$$

Which contradicts the fact that $\overline{\bar{P}}_{11}>0$. Then, $\overline{\bar{A}}_{22}$ is non singular.

The second step is to show that matrix $\left(\overline{\bar{A}}_{11}-\overline{\bar{A}}_{12} \overline{\bar{A}}_{22}^{-1} \overline{\bar{A}}_{21}\right)$ is stable.

For this, let us consider the transformed Lyapunov inequality

$$
\left(J \bar{A} J^{\top}\right)\left(J \bar{P} J^{\top}\right)\left(J \bar{A}^{\top} J^{\top}\right)-\left(J \bar{E} J^{-1}\right)\left(J \bar{P} J^{\top}\right)\left(J \bar{E}^{\top} J^{\top}\right)<0
$$

or similarly

$$
(\bullet)^{\top}\left(\left[\begin{array}{ll}
\overline{\bar{P}}_{11} & \overline{\bar{P}}_{12} \\
\overline{\bar{P}}_{21} & \bar{P}_{22}
\end{array}\right]\right)\left(\left[\begin{array}{ll}
\overline{\bar{A}}_{11}^{\top} & \overline{\bar{A}}_{21}^{\top} \\
\overline{\bar{A}}_{12}^{\top} & \overline{\bar{A}}_{22}^{\top}
\end{array}\right]\right)-\left[\begin{array}{cc}
\overline{\bar{P}}_{11} & 0 \\
0 & 0
\end{array}\right]<0 .
$$

Multiplying the right side of (29) by

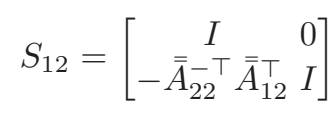

and the left side by its transpose, we get

$$
(\bullet)^{\top}\left(\left[\begin{array}{cc}
\overline{\bar{P}}_{11} & \overline{\bar{P}}_{12} \\
\overline{\bar{P}}_{21} & \bar{P}_{22}
\end{array}\right]\right)\left(\left[\begin{array}{cc}
\mathcal{A}^{\top} & \overline{\bar{A}}_{21}^{\top} \\
0 & \overline{\bar{A}}_{22}^{\top}
\end{array}\right]\right)-\left[\begin{array}{cc}
\overline{\bar{P}}_{11} & 0 \\
0 & 0
\end{array}\right]<0 .
$$

Note also that the last inequality can be written as follows

$$
(\bullet)^{\top}\left(S_{21}^{-\top}\left[\begin{array}{cc}
\overline{\bar{P}}_{11} & \overline{\bar{P}}_{12} \\
\overline{\bar{P}}_{21} & \bar{P}_{22}
\end{array}\right] S_{21}^{-1}\right)\left(S_{21}\left[\begin{array}{cc}
\mathcal{A}^{\top} & \overline{\bar{A}}_{21}^{\top} \\
0 & \bar{A}_{22}^{\top}
\end{array}\right]\right)-\left[\begin{array}{cc}
\overline{\bar{P}}_{11} & 0 \\
0 & 0
\end{array}\right]<0 .
$$

with

$$
S_{21}=\left[\begin{array}{cc}
I-\overline{\bar{A}}_{21}^{\top} \overline{\bar{A}}_{22}^{-\top} \\
0 & I
\end{array}\right]
$$

or equivalently

$$
(\bullet)^{\top}\left(S_{21}^{-\top}\left[\begin{array}{cc}
\overline{\bar{P}}_{11} & \overline{\bar{P}}_{12} \\
\overline{\bar{P}}_{21} & \overline{\bar{P}}_{22}
\end{array}\right] S_{21}^{-1}\right)\left(\left[\begin{array}{cc}
\mathcal{A}^{\top} & 0 \\
0 & \overline{\bar{A}}_{22}^{\top}
\end{array}\right]\right)-\left[\begin{array}{cc}
\overline{\bar{P}}_{11} & 0 \\
0 & 0
\end{array}\right]<0 .
$$

Notice that

$$
\begin{aligned}
& \left(S_{21}^{-\top}\left[\begin{array}{ll}
\overline{\bar{P}}_{11} & \overline{\bar{P}}_{12} \\
\overline{\bar{P}}_{21} & \overline{\bar{P}}_{22}
\end{array}\right] S_{21}^{-1}\right)=S_{21}^{-\top}\left[\begin{array}{lll}
\overline{\bar{P}}_{11} & \overline{\bar{P}}_{12}+\overline{\bar{P}}_{11} \overline{\bar{A}}^{\top} \overline{\bar{A}}_{22}^{-\top} \\
\overline{\bar{P}}_{21} & \overline{\bar{P}}_{22}+\overline{\bar{P}}_{21} \overline{\bar{A}}_{21}^{\top} \overline{\bar{A}}_{22}^{-\top}
\end{array}\right] \\
& =\left[\begin{array}{c}
\overline{\bar{P}}_{11} \overline{\bar{P}}_{12}+\overline{\bar{P}}_{11} \overline{\bar{A}}_{21}^{\top} \overline{\bar{A}}_{22}^{-\top} \\
\overline{\bar{P}}_{21}+\overline{\bar{A}}_{22}^{-1} \overline{\bar{A}}_{21} \overline{\bar{P}}_{11} \overline{\bar{P}}_{22}+\overline{\bar{P}}_{21} \overline{\bar{A}}_{21}^{\top} \overline{\bar{A}}_{22}^{-\top}+\overline{\bar{A}}_{22}^{-1} \overline{\bar{A}}_{21} \overline{\bar{P}}_{12}+\overline{\bar{A}}_{22}^{-1} \overline{\bar{A}}_{21} \overline{\bar{P}}_{11} \overline{\bar{A}}_{21}^{\top} \overline{\bar{A}}_{22}^{-\top}
\end{array}\right]
\end{aligned}
$$


which shows that (31) can be equivalently written as follows:

$$
(\bullet)^{\top}\left(\left[\begin{array}{cc}
\overline{\bar{P}}_{11} & * \\
* & *
\end{array}\right]\right)\left(\left[\begin{array}{cc}
\mathcal{A}^{\top} & 0 \\
0 & \overline{\bar{A}}_{22}^{\top}
\end{array}\right]\right)-\left[\begin{array}{cc}
\overline{\bar{P}}_{11} & 0 \\
0 & 0
\end{array}\right]<0 .
$$

where $(*)$ denotes expressions that are irrelevant.

From (32) we get easily

$$
\mathcal{A} \overline{\bar{P}}_{11} \mathcal{A}^{\top}-\overline{\bar{P}}_{11}<0
$$

which states the stability of matrix $\mathcal{A}$, having in mind the positivity of $\overline{\bar{P}}_{11}$. This concludes the proof of Theorem 2

\section{Admissibilization of the 2-D system}

In this section, we first look for a strict inequality condition to check the stability of the system, to replace the two conditions (17) and (18).

Theorem 3 The $2 D$ singular system described by the Roesser model is admissible for a given $\beta>1$, if and only if there exist matrices $P$ and $X$ such that:

$$
\left[\begin{array}{cc}
-E P E^{\top} & Z^{\top} \\
Z & P
\end{array}\right]+\operatorname{Sym}\left\{\left[\begin{array}{c}
A \\
-I
\end{array}\right] X\left[U^{-\top}-\beta V^{\top}\right]\right\}<0
$$

Proof

Notice that condition (18) can be written, for $Z=0$, as follows

$$
\left[\begin{array}{ll}
I & A
\end{array}\right]\left[\begin{array}{cc}
-E P E^{\top} & Z^{\top} \\
Z & P
\end{array}\right]\left[\begin{array}{c}
I \\
A^{\top}
\end{array}\right]<0
$$

Condition (17) can be written equivalently as follows

$$
\left[\begin{array}{ll}
I & I
\end{array}\right] \underbrace{\left[\begin{array}{cc}
-E P E^{\top} & -E^{\dagger} Y \\
-\left(E^{\dagger} Y\right)^{\top} & 0
\end{array}\right]}_{\Phi_{1}}\left[\begin{array}{l}
I \\
I
\end{array}\right]<0
$$

which is in a similar form as:

$$
\left[\beta U V^{-1}\right]\left[\begin{array}{cc}
-E P E^{\top} & Z^{\top} \\
Z & P
\end{array}\right]\left[\begin{array}{l}
\beta U^{\top} \\
V^{-\top}
\end{array}\right]<0
$$

where $Z$ is a matrix that has to be selected to recover the regularity and the stability of the system. The 22-block of the multiplier $\Phi_{1}$ has been changed from zero to $P$ in order to write (17)-(18) respectively as (34)-(35).

According to the projection lemma [22, Theorem 2.3.12], conditions (35)(36) are equivalent to the existence of a matrix $X$ of appropriate dimension such as (34) holds. 
If we expand (36) we get

$$
-\beta^{2} U E P E^{\top} U^{\top}+V^{-1} P V^{-\top}+\operatorname{Sym}\left\{\beta U Z^{\top} V^{-\top}\right\}<0
$$

or equivalently

$$
\begin{gathered}
-\beta^{2}\left(J^{\top} U E V J\right)\left(J^{\top} V^{-1} P V^{-\top} J\right)\left(J^{\top} V^{\top} E^{\top} U^{\top} J\right) \\
+J^{\top} V^{-1} P V^{-\top} J+\operatorname{Sym}\left\{\beta J^{\top} U Z^{\top} V^{-\top} J\right\}<0 .
\end{gathered}
$$

A simple way to satisfy the condition above is to choose $Z$ in such a way that

$$
\operatorname{Sym}\left\{\beta J^{\top} U Z^{\top} V^{-\top} J\right\}=\left[\begin{array}{l}
0 * \\
* *
\end{array}\right]
$$

where $(*)$ are irrelevant expressions. This yields

$$
-\beta^{2}\left[\begin{array}{cc}
\overline{\bar{P}}_{11} & 0 \\
0 & 0
\end{array}\right]+\left[\begin{array}{cc}
\overline{\bar{P}}_{11} & \overline{\bar{P}}_{12} \\
\overline{\bar{P}}_{21} & \overline{\bar{P}}_{22}
\end{array}\right]+\left[\begin{array}{l}
0 * \\
* *
\end{array}\right]<0
$$

which implies that $\left(1-\beta^{2}\right) \overline{\bar{P}}_{11}<0$, and since $\beta>1$, then $\overline{\bar{P}}_{11}$ is strictly positive definite.

If we expand (35) and after some appropriate manipulations, we get

$$
\begin{gathered}
-\left(J^{\top} U E V J\right)\left(J^{\top} V^{-1} P V^{-\top} J\right)\left(J^{\top} V^{\top} E^{\top} U^{\top} J\right) \\
+\left(J^{\top} U A V J\right)\left(J^{\top} V^{-1} P V^{-\top} J\right)\left(J^{\top} V^{\top} A^{\top} U^{\top} J\right) \\
+\operatorname{Sym}\left\{\left(J^{\top} U A V J\right)\left(J^{\top} V^{-1} Z U^{\top} J\right)\right\}<0,
\end{gathered}
$$

or more explicitly

$$
\begin{aligned}
& -\left[\begin{array}{cc}
\overline{\bar{P}}_{11} & 0 \\
0 & 0
\end{array}\right]+\left[\begin{array}{cc}
\overline{\bar{A}}_{11} & \overline{\bar{A}}_{12} \\
\overline{\bar{A}}_{21} & \overline{\bar{A}}_{22}
\end{array}\right]\left[\begin{array}{cc}
\overline{\bar{P}}_{11} & \overline{\bar{P}}_{12} \\
\overline{\bar{P}}_{21} & \overline{\bar{P}}_{22}
\end{array}\right]\left[\begin{array}{cc}
\overline{\bar{A}}_{11} & \overline{\bar{A}}_{12} \\
\bar{A}_{21} & \overline{\bar{A}}_{22}
\end{array}\right]^{\top} \\
& +\operatorname{Sym}\left\{\left(J^{\top} U A V J\right)\left(J^{\top} V^{-1} Z U^{\top} J\right)\right\}<0 .
\end{aligned}
$$

If matrix $J^{\top} V^{-1} Z U^{\top} J$ is partitioned as follows:

$$
J^{\top} U Z^{\top} V^{-\top} J=\left[\begin{array}{cc}
0 & 0 \\
* & \overline{\bar{Z}}_{22}
\end{array}\right]
$$

we get

$$
\begin{aligned}
& -\left[\begin{array}{cr}
\overline{\bar{P}}_{11} & 0 \\
0 & 0
\end{array}\right]+\left[\begin{array}{cc}
\overline{\bar{A}}_{11} & \overline{\bar{A}}_{12} \\
\overline{\bar{A}}_{21} & \overline{\bar{A}}_{22}
\end{array}\right]\left[\begin{array}{cc}
\overline{\bar{P}}_{11} & \overline{\bar{P}}_{12} \\
\overline{\bar{P}}_{21} & \overline{\bar{P}}_{22}
\end{array}\right]\left[\begin{array}{cc}
\overline{\bar{A}}_{11} & \overline{\bar{A}}_{12} \\
\overline{\bar{A}}_{21} & \overline{\bar{A}}_{22}
\end{array}\right]^{\top} \\
& +\operatorname{Sym}\left\{\left[\begin{array}{ll}
\overline{\bar{A}}_{11} & \overline{\bar{A}}_{12} \\
\overline{\bar{A}}_{21} & \overline{\bar{A}}_{22}
\end{array}\right]\left[\begin{array}{cc}
0 & 0 \\
* & \overline{\bar{Z}}_{22}
\end{array}\right]\right\}<0
\end{aligned}
$$


and taking account of (26), we get

$$
\begin{aligned}
-\left[\begin{array}{cc}
\overline{\bar{P}}_{11} & 0 \\
0 & 0
\end{array}\right]+ & {\left[\begin{array}{ll}
L_{11} \overline{\bar{P}}_{11} L_{11}^{\top}+\overline{\bar{A}}_{12} \hat{P}_{22} \overline{\bar{A}}_{21}^{\top} & L_{11} \overline{\bar{P}}_{11} L_{12}^{\top}+\overline{\bar{A}}_{12} \hat{P}_{22} \overline{\bar{A}}_{22}^{\top} \\
L_{12} \overline{\bar{P}}_{11} L_{11}^{\top}+\overline{\bar{A}}_{22} \hat{P}_{22} \overline{\bar{A}}_{21}^{\top} & L_{12} \overline{\bar{P}}_{11} L_{12}^{\top}+\overline{\bar{A}}_{22} \hat{P}_{22} \overline{\bar{A}}_{22}^{\top}
\end{array}\right] } \\
& +\operatorname{Sym}\left\{\left[\begin{array}{ll}
\overline{\bar{A}}_{11} & \overline{\bar{A}}_{12} \\
\overline{\bar{A}}_{21} & \bar{A}_{22}
\end{array}\right]\left[\begin{array}{ll}
0 & 0 \\
* & \overline{\bar{Z}}_{22}
\end{array}\right]\right\}<0
\end{aligned}
$$

which yields

$$
L_{12} \overline{\bar{P}}_{11} L_{12}^{\top}+\overline{\bar{A}}_{22} \hat{P_{22}} \overline{\bar{A}}_{22}^{\top}+\operatorname{Sym}\left\{\overline{\bar{A}}_{22} \overline{\bar{Z}}_{22}\right\}<0
$$

an inequality that asserts that $\overline{\bar{A}}_{22}^{\top}$ is non singular.

Going back to (38) and performing the same manipulations used to obtain (31), we get

$$
\begin{aligned}
(\bullet)^{\top}\left(S_{21}^{-\top}\right. & {\left.\left[\begin{array}{cc}
\overline{\bar{P}}_{11} & \overline{\bar{P}}_{12} \\
\overline{\bar{P}}_{21} & \overline{\bar{P}}_{22}
\end{array}\right] S_{21}^{-1}\right)\left(\left[\begin{array}{cc}
\mathcal{A}^{\top} & 0 \\
0 & \overline{\bar{A}}_{22}^{\top}
\end{array}\right]\right) } \\
& -\left[\begin{array}{cc}
\overline{\bar{P}}_{11} & 0 \\
0 & 0
\end{array}\right]+\operatorname{Sym}\left\{\left[\begin{array}{cc}
\mathcal{A} & 0 \\
0 & \overline{\bar{A}}_{22}
\end{array}\right]\left[\begin{array}{ll}
0 & 0 \\
* *
\end{array}\right]\right\}<0 .
\end{aligned}
$$

The top left matrix in inequality (39) is

$$
\mathcal{A} \overline{\bar{P}}_{11} \mathcal{A}^{\top}-\overline{\bar{P}}_{11}<0
$$

which asserts that $\mathcal{A}$ is stable.

One can notice that (35) and (36) can be recovered using backward the same argument above which results in (34) and the equivalence is proved.

Let us now look for a matrix $Z$ which satisfies condition (37). If we choose a matrix $Z$ as follows:

$$
Z=\left(V J\left(I-J V^{\top} E^{\top} U^{\top} J^{\top}\right) J^{\top} U^{-\top}\right)
$$

then we get

$$
\begin{aligned}
\left(J^{\top} V^{-1} Z U^{\top} J\right) & =I-J V^{\top} E^{\top} U^{\top} J^{\top} \\
& =\left[\begin{array}{ll}
0 & 0 \\
0 & \overline{\bar{Z}}_{22}
\end{array}\right]
\end{aligned}
$$

which shows effectively that $Z$ complies with condition (37). 
5.1 Stabilization of 2D singular systems

In this subsection, we will deal with the problem of stabilization by state feedback for the $2 \mathrm{D}$ singular system given by:

$$
E\left[\begin{array}{l}
x^{h}(i+1, j) \\
x^{v}(i, j+1)
\end{array}\right]=A\left[\begin{array}{l}
x^{h}(i, j) \\
x^{v}(i, j)
\end{array}\right]+B u(i, j)
$$

where $u(i, j) \in \mathbb{R}^{m}$ is the control input.

The control law given by a state feedback is then

$$
u(i, j)=K\left[\begin{array}{l}
x^{h}(i, j) \\
x^{v}(i, j)
\end{array}\right]
$$

where the gain $K$, of appropriate dimension, is computed in a way that the singular closed loop system is admissible.

Theorem 4 The closed loop singular system is admissible with the state feedback

$$
K=G X^{-1}
$$

for a given $\beta \in \mathbb{R}$, with $\beta>1$, if and only if the following LMI is feasible in the variables $X, P, G$ :

$$
\left[\begin{array}{cc}
-E P E^{\top} & Z^{\top} \\
Z & P
\end{array}\right]+\operatorname{Sym}\left\{\left[\begin{array}{c}
A X+B G \\
-X
\end{array}\right]\left[U^{-\top}-\beta V^{\top}\right]\right\}<0
$$

Proof

The closed loop 2D singular system is admissible if and only if there exist $X$, $P$, and a state feedback $K$ satisfying the following LMI

$$
\left[\begin{array}{cc}
-E P E^{\top} & Z^{\top} \\
Z & P
\end{array}\right]+\operatorname{Sym}\left\{\left[\begin{array}{c}
A+B K \\
-I
\end{array}\right] X\left[U^{-\top}-\beta V^{\top}\right]\right\}<0
$$

for a given $\beta>1$. Thus, it is clear that a change of variable $G=K X$ yields condition (44) which completes the proof.

\section{Example}

To highlight the effectiveness of the proposed approach, a numerical example is now developed. Consider the 2D SRM in (40) with the following system 
matrices:

$$
\begin{aligned}
A & =\left[\begin{array}{ccccc}
0.0400 & -0.6800 & 0.7600 & -1.4480 & 2.2320 \\
0.1160 & -0.7720 & 0.6840 & -0.6000 & 0.6800 \\
-0.4720 & 1.9440 & 0.5520 & -0.2800 & 0.6400 \\
-0.8400 & -0.9160 & 0.6800 & 0.1200 & 0.4000 \\
0.4000 & 0.6000 & 0.5520 & 0.1200 & 0.6400
\end{array}\right] \\
E & =\left[\begin{array}{lllll}
1 & 0 & 0 & 0 & 0 \\
0 & 1 & 0 & 0 & 0 \\
0 & 0 & 0 & 0 & 0 \\
0 & 0 & 0 & 1 & 0 \\
0 & 0 & 0 & 0 & 0
\end{array}\right], \\
B & =\left[\begin{array}{lllll}
1.00 & -0.10 & 2.20 & 0.50 & 0.70
\end{array}\right]^{\top} .
\end{aligned}
$$

The open loop system is not regular, with the corresponding matrix $\overline{\bar{A}}_{22}$ not invertible.

By solving the LMI (43), with the design parameter $\beta=2$ the following feasible solution can be found:

$$
\begin{aligned}
P & =10^{3}\left[\begin{array}{ccccc}
0.2615 & -0.2609 & 0.1178 & 0 & 0 \\
-0.2609 & 0.2604 & -0.1254 & 0 & 0 \\
0.1178 & -0.1254 & -1.2129 & 0 & 0 \\
0 & 0 & 0 & 0.0076 & 0.0143 \\
0 & 0 & 0 & 0.0143 & -0.4475
\end{array}\right] \\
X & =\left[\begin{array}{ccccc}
-104.1804 & 81.3792 & -120.3466 & -15.3932 & -77.3744 \\
90.4095 & -97.7882 & 154.1874 & 11.8161 & 27.7099 \\
31.7315 & -69.5319 & 231.2352 & -0.1020 & -32.2636 \\
30.7065 & -0.4232 & 4.4671 & -38.4950 & 10.5835 \\
83.6472 & -51.1011 & 64.5327 & -17.0386 & 52.9031
\end{array}\right] \\
G & =\left[\begin{array}{lllll}
-95.0569 & 94.6840-282.8348 & -12.5036 & -64.8997
\end{array}\right] \\
K & =\left[\begin{array}{lllll}
0.3765 & 2.2150-1.6297 & 2.3162 & -3.2936
\end{array}\right]
\end{aligned}
$$

Some simulated results are now presented, with null boundary values for $i>250$ or $j>250$. To facilitate the visualitation, we plot in Figure 6 the evolution of the two following quantities, which correspond to the norm of the generalized vector along the diagonal $i+j=k$ :

$$
\begin{aligned}
X^{h}(k) & =\sum_{i=0}^{k}\left\|x^{h}(i, k-i)\right\|^{2} \\
X^{v}(k) & =\sum_{i=0}^{k}\left\|x^{v}(i, k-i)\right\|^{2}
\end{aligned}
$$

This makes possible to compress the evolution of the $2 \mathrm{D}$ system using $1 \mathrm{D}$ plots: It can be seen that they go to zero as $k$ goes to infinity, indicating that the system is stable. 


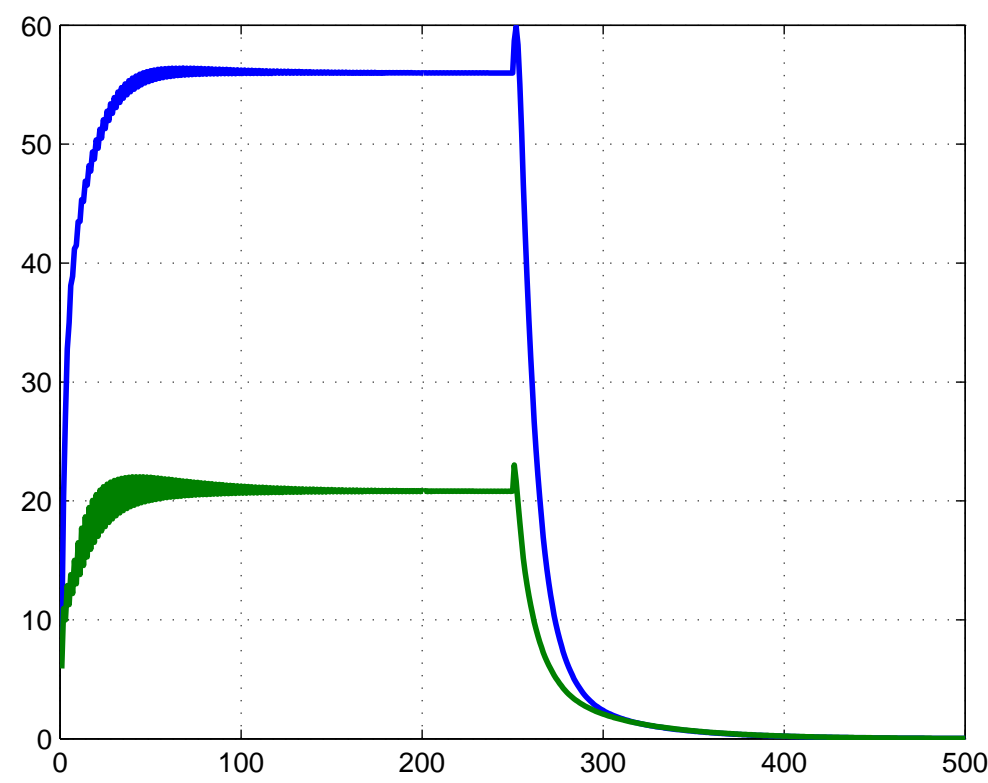

The variations of $\left\|X^{h}\right\|$ and $\left\|X^{v}\right\|$

\section{Conclusion}

In this paper, we consider the stabilization of a class of $2 \mathrm{D}$ singular systems, expressed in a separated Roesser form; that is, with the singular matrix $E$ partitioned as a two blocks diagonal matrix, where the blocks correspond to the horizontal and vertical directions. These separated Roesser form allows to derive algebraic equations on each direction, making possible to rewrite the regularity condition as the non singularity of a certain matrix. A sufficient stability condition is then established, which makes possible to check the admissibility of the $2 \mathrm{D}$ singular system. To design feedback controllers, a sufficient but strict LMI condition is established and then used in the design of a state feedback controller.

A numeric example is given at the end of the paper to illustrate the effectiveness of the proposed approach, showing how it is possible to design controllers that provides admissibility for the closed loop system. As further work, the proposed approach could be extended to multidimensional systems or systems with constraints.

\section{References}

1. Boudellioua, M.S., Galkowski, K.: Reduction of wave linear repetitive processes to 2-d singular state space form. In: 23rd International Symposium on Mathematical Theory of 
Networks and Systems, pp. 677-682. Hong Kong University of Science and Technology, Hong Kong (2018)

2. Boudellioua, M.S., Galkowski, K., Rogers, E.: On the connection between discrete linear repetitive processes and 2-d discrete linear systems. Multidimensional Systems and Signal Processing 28, 341-351 (2017). DOI 10.1007/s11045-016-0454-8

3. Cai, C., Zou, Y.: A note on the internal stability for 2-D singular discrete systems. Multidimensional Systems and Signal Processing 15(2), 197-204 (2004)

4. Chaabane, M., Bachelier, O., Souissi, M., Mehdi, D.: Stability and stabilization of continuous descriptor systems: an lmi approach. Mathematical Problems in Engineering $2006(2006)$

5. Chaabane, M., Tadeo, F., Mehdi, D., Souissi, M.: Robust admissibilization of descriptor systems by static output-feedback: an lmi approach. Mathematical Problems in Engineering 2011 (2011)

6. Chen, S.F.: Stability analysis and stabilization of $2 \mathrm{D}$ singular Roesser models. Applied Mathematics and Computation 250, 779-791 (2015)

7. Duan, G.R.: Analysis and design of descriptor linear systems, vol. 23. Springer Science and Business Media (2010)

8. Galkowski, K.: A perspective on singularity in 2d linear systems. Multidimensional Systems and Signal Processing 11(1), 83-108 (2000)

9. Galkowski, K., Lam, J., Xu, S., Lin, Z.: LMI approach to state-feedback stabilization of multidimensional systems. International Journal of Control 76(14), 1428-1436 (2003)

10. Ghamgui, M., Mehdi, D., Bachelier, O., Chaabane, M.: Stability of 2D discrete takagisugeno systems. CSSP 36(6), 2256-2274 (2017)

11. Ghamgui, M., Yeganefar, N., Bachelier, O., Mehdi, D.: Stability and stabilization of $2 \mathrm{D}$ continuous state-delayed systems. In: Decision and Control and European Control Conference (CDC-ECC), 2011 50th IEEE Conference on, pp. 1878-1883. IEEE (2011)

12. Hinamoto, T.: Stability of 2-D discrete systems described by the Fornasini-Marchesini second model. IEEE Transactions on Circuits and Systems I: Fundamental Theory and Applications 44(3), 254-257 (1997)

13. Kaczorek, T. (ed.): Two-Dimensional Linear Systems, Lecture Notes in Control and Information Science, vol. 68. Springer-Verlag, New York (1985)

14. Kririm, S., Hmamed, A., Tadeo, F.: Robust $H_{\infty}$ filtering for uncertain 2D singular Roesser models. Circuits, Systems, and Signal Processing 34(7), 2213-2235 (2015)

15. Liu, D.: Lyapunov stability of two-dimensional digital filters with overflow nonlinearities. IEEE Transactions on Circuits and Systems I: Fundamental Theory and Applications 45(5), 574-577 (1998)

16. Liu, D., Michel, A.N.: Stability analysis of state-space realizations for two-dimensional filters with overflow nonlinearities. IEEE Transactions on Circuits and Systems I: Fundamental Theory and Applications 41, 127-137 (1994)

17. Lu, W.S.: Two-dimensional digital filters, vol. 80. CRC Press (1992)

18. Paszke, W., Lam, J., Galkowski, K., Xu, S., Lin, Z.: Robust stability and stabilization of 2D discrete state-delayed systems. Systems and Control Letters 51, 277-291 (2004)

19. Rejichi, O., Bachelier, O., Chaabane, M., Mehdi, D.: Admissibility and state feedback admissibilization of discrete singular systems: An lmi approach. In: Control and Automation, 2008 16th Mediterranean Conference on, pp. 1022-1027. IEEE (2008)

20. Saadni, S.M., Chaabane, M., Mehdi, D., Bachelier, O.: Robust stability and stabilization of a class of singular systems with multiple time-varying delays. IFAC Proceedings Volumes 38(1), 161-166 (2005)

21. Shaker, H.R., Shaker, F.: Lyapunov stability for continuous-time multidimensional nonlinear systems. Nonlinear Dynamics 75(4), 717-724 (2014)

22. Skelton, R.E., K., T.I., Grigoriadis: A Unified Algebraic Approach to Linear Control Design. Taylor-Francis, Bristol-USA (1998)

23. Tiwari, M., Dhawan, A.: A survey on the stability of 2D discrete systems described by fornasini-marchesini second model. Circuits and Systems 3(1), 17 (2012)

24. Wang, L., Wang, W., Chen, W.: Delay-dependent stability for 2D singular systems with state-varying delay in Roesser model: An lmi approach. In: Control Conference (CCC), 2014 33rd Chinese, pp. 6074-6079. IEEE (2014)

25. Xiang, Z., Huang, S.: Stability analysis and stabilization of discrete-time 2D switched systems. Circuits, Systems, and Signal Processing 32(1), 401-414 (2013) 
26. Xu, H., Zou, Y.: $H_{\infty}$ control for $2 \mathrm{D}$ singular delayed systems. International Journal of Systems Science 42(4), 609-619 (2011)

27. Xu, H., Zou, Y., Xu, S., Lam, J.: Bounded real lemma and robust $H_{\infty}$ control of $2 \mathrm{D}$ singular Roesser models. Systems and control letters 54(4), 339-346 (2005)

28. Xu, S., Lam, J.: $H_{\infty}$ model reduction for discrete-time singular systems. Systems and control letters 48(2), 121-133 (2003)

29. Zou, Y., Campbell, S.L.: The jump behavior and stability analysis for 2D singular systems. Multidimensional Systems and Signal Processing 11(4), 339-358 (2000)

30. Zou, Y., Xu, H.: Duality of 2-d singular systems of roesser models. Journal of Control Theory and Applications 5, 37-41 (2007)

31. Zou, Y., Xu, H., Wang, W.: Stability for two-dimensional singular discrete systems described by general model. Multidimensional Systems and Signal Processing 19(2), 219-229 (2008) 